\title{
Estrategias para la mejora del desempeño del Profesor Asociado en la universidad
}

\author{
Beatriz Garcia-Ortega ${ }^{a}$, Javier Galan-Cubillo ${ }^{b}$, Blanca de-Miguel-Molina ${ }^{c}$ \\ ${ }^{a}$ Departamento de Organización de Empresas, Universitat Politècnica de València, Valencia, Spain, \\ beagaror@doctor.upv.es; ${ }^{b}$ Programa Doctoral en Administración de Empresas, Universitat \\ Politècnica de València, Valencia, Spain, jagacu@doctor.upv.es; 'Departmento de Organización de \\ Empresas, Universitat Politècnica de València, Valencia, Spain, bdemigu@omp.upv.es
}

\begin{abstract}
Resumen
El Profesor Asociado universitario está llamado a contribuir con su experiencia al equilibrio entre teoría y práctica y a conseguir una mayor conexión entre el mundo académico y el laboral y social. Sin embargo, existe una controversia en torno al uso que se da a esta figura, que supone ya más del $20 \%$ de los docentes universitarios en España. A pesar de ello, hay una escasez de publicaciones que se centren en las estrategias para mejorar su desempeño. A partir de las publicaciones consultadas y que son parte del marco teórico de referencia, este artículo lleva a cabo un análisis crítico y constructivo de posibles estrategias y propuestas innovadoras para la mejora del desempeño de este colectivo, con el objeto de promover la discusión entre los académicos en este ámbito.
\end{abstract}

Palabras clave: profesor asociado, docencia universitaria, mejora del desempeño

\footnotetext{
Abstract

The University Part-time Assistant Professor is called to contribute with his experience to the balance between theory and practice and to achieve a greater connection between the academic world and the labor and social world. However, there is a controversy regarding the use given to this figure, which already represents more than 20\% of university professors in Spain. Despite this, there is a paucity of publications that focus on strategies to improve their performance. Starting from the consulted publications, which are part of our theorical framework of reference, this article carries out a critical and constructive analysis of possible strategies and innovative
} 
proposals for improving the performance of this group, in order to promote discussion among the academics in this field.

Keywords: associate professor, university teaching, performance improvement

\section{Introducción}

De acuerdo al informe de Datos y Cifras del Sistema Universitario Español (Ministerio de Ciencia, Innovación y Universidades, 2019), más del $20 \%$ de los docentes son ya profesores asociados, y suponen más del $40 \%$ del personal docente e investigador contratado cada año.

La figura del Profesor Asociado se creó en la Ley de Reforma Universitaria (LRU), de 25 de Agosto de 1983, art ${ }^{\circ} .33$. Esta figura se concibió entre otros para incorporar el espíritu de la empresa, contribuir con su experiencia al equilibrio entre teoría y práctica y conseguir una mayor conexión entre el mundo académico y el laboral y social, pero existen voces que apuntan a una perversión de esta figura y a su precarización, especialmente tras el estallido de la crisis económica y los recortes en la tasa de reposición desde el año 2010 (SánchezCaballero, 2019). De hecho, esta figura ha supuesto una fuente de litigios, cuestionando en algunos casos si se tratan realmente de profesionales de reconocido prestigio, así como el supuesto carácter abusivo y/o fraudulento de las contrataciones temporales reiteradas y sucesivas para cubrir necesidades permanentes de la universidad (i Gené, 2018). Además, la literatura se inclina por que la proliferación de esta figura puede afectar negativamente al logro en la Universidad (López Damián et al., 2016).

A pesar del importante papel al que está llamado el Profesor Asociado en la universidad, su creciente peso en las universidades españolas y su cuestionamiento, detectamos una escasez de trabajos académicos que se centren en las posibles estrategias para mejorar su desempeño y la aportación de ideas innovadoras para su consideración por parte de la comunidad universitaria.

En este artículo se pretende realizar un análisis crítico y constructivo de los aspectos y estrategias que pueden contribuir a la mejora del papel del Profesor Asociado en el ámbito de la formación universitaria, con el apoyo de la literatura y a partir de la experiencia profesional y docente universitaria y diferentes perspectivas de los autores, uno de ellos Profesor Asociado, otro Profesor colaborador y el tercero Profesor titular. 


\section{El Profesor Asociado, marco legislativo y contexto actual}

La Real Academia de la Lengua define el Profesor Asociado como 'Profesor que, desarrollando regularmente su actividad fuera de la Universidad y siendo especialista de reconocida competencia, es contratado de forma temporal y con dedicación parcial para que aporte sus conocimientos y experiencia profesional en la Universidad.'

Dentro del Boletín Oficial del Estado (BOE), en su documento con referencia BOE-A2007-7786, en vigor a partir del 03/05/2007, establece en su Artículo 53 lo siguiente:

La contratación de Profesoras y Profesores Asociados se ajustará a las siguientes reglas:

a) El contrato se podrá celebrar con especialistas de reconocida competencia que acrediten ejercer su actividad profesional fuera del ámbito académico universitario.

b) La finalidad del contrato será la de desarrollar tareas docentes a través de las que aporten sus conocimientos y experiencia profesionales a la universidad.

c) El contrato será de carácter temporal y con dedicación a tiempo parcial.

d) La duración del contrato será trimestral, semestral o anual, y se podrá renovar por periodos de igual duración, siempre que se siga acreditando el ejercicio de la actividad profesional fuera del ámbito académico universitario.»

El Profesor Asociado constituye pues un puente entre la universidad y la empresa privada, contribuye al equilibrio entre teoría y práctica, permite una conexión de los alumnos con su sector y área de especialización y favorece su futura integración en el ámbito laboral y social.

El trabajo de Profesor Asociado tiene en teoría el carácter de tiempo parcial, si bien en algunas situaciones y países ha supuesto una puerta de entrada para llegar a trabajar a tiempo completo (López Damián et al., 2016; i Gené, 2018), o bien para atender necesidades permanentes de la universidad (i Gené, 2018), lo que entra en contradicción con su propia condición de contrato a tiempo parcial.

Algunos estudios indican que el Profesor Asociado entre otras modalidades permite un ahorro de costes y una mayor flexibilidad (Ehrenberg, 2012; Crosier et al., 2017). Otros vinculan a los profesores a tiempo parcial como un grupo de bajo compromiso laboral (Umbach, 2007). Vimos además en la introducción el cuestionamiento de la utilización de este tipo de contrato, y que existen estudios que vinculan el aumento del porcentaje de estos profesores con la disminución de la calidad universitaria. 
El profesor universitario tiene como funciones principales la investigación, la docencia y la difusión de información (Becher, 2001). Al igual que cualquier profesor a tiempo completo, el profesor asociado está adscrito a una educación superior y, además de cumplir con la difusión del conocimiento a través de la docencia (López Damián et al., 2016), la investigación entraría dentro de sus posibles funciones, a pesar de que el BOE se refiere únicamente a 'tareas docentes'.

Paradójicamente, el sistema de docencia establecido en la universidad española supone una sobrecarga para el profesorado a tiempo completo que los profesores no ven recompensada (González González et al., 2014), de tal modo que en la práctica la docencia es infravalorada (Carreras Barnés et al., 2006) en relación a la investigación. Para el profesor asociado, sin embargo, la docencia suele ser una fuente de recompensa y satisfacción personal, al tener con frecuencia un fuerte componente vocacional y compaginarse con la práctica profesional (Cladellas-Pros et al., 2018). Su contribución docente permite al resto de profesorado centrarse más en la investigación y maximizar su productividad (Baldwin y Chronister, 2001).

\section{Discusión de estrategias de mejora en el desempeño del Profesor Asociado}

Cuando entramos a valorar el desempeño de un profesor y cómo mejorarlo, tenemos que hacerlo desde la perspectiva de sus competencias. Es necesario contemplar las competencias profesionales que precisa el profesor universitario para el desarrollo de sus funciones (Más-Torelló, 2011). De su recopilación de acepciones, a nuestro objeto entendemos como competencias profesionales la acumulación de conocimientos y experiencias y la capacidad de aplicarlos, en continua revisión de acuerdo a sus nuevas experiencias y aprendizajes, de tal modo que permite un nivel de desempeño y solucionar situaciones, problemas y retos que surgen durante el ejercicio de la profesión.

Partiendo de la definición, funciones y aportaciones que se esperan del Profesor Asociado expuestas, y con el apoyo de la literatura, pasamos a continuación a reunir diferentes estrategias que pueden contribuir a la mejora de su desempeño.

\subsection{Protocolo de integración y tutorización}

El proceso de integración en la comunidad universitaria es uno de los primeros aspectos a considerar. Para un profesional del sector privado que haya estado fuera del ámbito universitario por un tiempo, los procedimientos e incluso los detalles que pueden resultar más evidentes son ajenos y resultan una barrera a la hora de una incorporación efectiva. Es 
frecuente que el proceso de integración se produzca de un modo asistemático y accidental, como una carrera de obstáculos, que depende de la buena voluntad de sus compañeros docentes y resto de personal universitario. Un manual de acogida o de bienvenida, unido a la figura de un tutor, se presentan como herramientas facilitadoras del proceso de integración.

El tutor puede guiar y monitorizar al profesor asociado especialmente en su primer contacto y primeros pasos en la docencia universitaria o al incorporarse a una nueva universidad. Entre otros puede introducirle en los aspectos operativos básicos, manejo de las herramientas informáticas, modular la asunción progresiva de responsabilidades, además de suponer un nexo con el resto de la comunidad universitaria, ayudar en la gestión del contexto institucional y fomentar su sentido de pertenencia. En esta línea, algunas universidades y departamentos desarrollan planes de acompañamiento para sus nuevos docentes (Más-Torelló, 2011). Una herramienta de ayuda es la creación de un modelo de simulación donde se visualizan de forma gráfica las necesidades, experiencia, preparación, intereses, capacidades y personalidades en el contexto de la Universidad que permiten la mejora de la competitividad del profesor en el proceso de enseñanza y aprendizaje (OsunaMillan et al., 2020).

\subsection{Cruzar competencias del profesor con características de la asignatura. La importancia de los criterios de selección}

Como primera consideración, la falta de dominio de la asignatura por parte de un profesor asociado genera una situación de inseguridad (Borrero y Losada, 2012). Hay que tratar de adecuar el perfil del profesor a los objetivos de la asignatura. En este sentido se plantean dos problemas principales. Por un lado, la figura de profesor asociado resulta tentadora a la hora de cubrir huecos o completar cuadros cuya casación resulta de gran complejidad. Por otro lado, se puede dar en ocasiones un cierto intrusismo en el sentido de supuestos profesionales que modifican su currículum para aparecer aptos para un puesto, por ejemplo, tratando de acreditar una actividad o unas funciones que realmente no realizan. En la actual legislación ya se eliminó con que desarrollen 'normalmente' su actividad fuera de la universidad, sino que debe acreditarse 'ejercer su actividad profesional fuera del ámbito académico universitario’ (i Gené, 2018). Se ha de estar vigilante y se deben reforzar los mecanismos que permitan identificar y discriminar perfiles, contrastando la veracidad y adecuación al perfil requerido.

Es muy importante además la ponderación de méritos, donde dada la idiosincrasia del profesor asociado se debe primar entre otros su competencia profesional relacionada con la asignatura (Más-Torelló, 2011) y la adecuación de su perfil por encima de otras 
consideraciones que pueden ser también relevantes, pero no tan decisivas en la calidad de la docencia.

\subsection{Compatibilidad con su actividad principal}

En muchas ocasiones resulta difícil encontrar al profesor asociado que encaje con los requisitos requeridos. Una vez esto sucede, el profesor asociado tiene parte de su jornada ya comprometida por su propia actividad laboral. La adaptación de los horarios en la medida de lo posible resulta fundamental para priorizar la asignación de asignaturas en función de las competencias por encima de necesidades logísticas. Ocurre que, debido a situaciones de emergencia para cubrir puestos o completar los Planes de Ordenación Docentes, no existe apenas margen de maniobra para tratar de asignar y encajar al profesional más idóneo para la asignatura y se pasa al siguiente de la lista.

\subsection{Involucración y participación crítica en las guías docentes y en el método didáctico}

La participación del profesional profesor asociado en las guías docentes y contenidos de las asignaturas puede resultar muy enriquecedor. El profesor asociado aporta su experiencia y punto de vista profesional, puede detectar lagunas o necesidad de actualización en los temarios o puntos donde se requiere mayor énfasis desde la perspectiva de las necesidades presentes y futuras en la empresa. También puede aportar en relación al método didáctico, dotarlo de un carácter más práctico o aplicar estrategias de formación de éxito de acuerdo a su experiencia en el ámbito privado. En este aspecto, y conviniendo que no hay un solo camino para llegar a la meta en cuanto a prácticas docentes, es valorable cierta flexibilidad a la hora de establecer el método docente en el que el profesor asociado se encuentre más cómodo.

\subsection{Docencia mixta}

La docencia mixta, donde un profesor titular y un asociado imparten una asignatura conjuntamente, combinando la parte teórica y la práctica, puede generar una sinergia y ofrecer el valor añadido del aprendizaje mutuo del propio profesorado. Dicha combinación también puede plantearse de forma no simultánea. Esta estrategia enriquece los contenidos y amplía la perspectiva del alumnado. 


\subsection{Formación docente continua}

Se trataría de fomentar un programa de cursos específicos orientados a este perfil, que le permita formarse en competencias pedagógicas, comunicativas y curriculares de acuerdo a los objetivos docentes de la Universidad, de modo que el profesional pueda alinearse con los mismos en su actividad, así como de su especialización. Informes como el de Eurydice abogan por este tipo de formación continua (Eurydice, 2004).

\subsection{Involucración en la investigación}

La Declaración de Bolonia de 1999 establece entre sus objetivos la promoción de la integración de la formación e investigación del profesorado universitario, mientras que la Carta Magna de las Universidades Europeas de 2001 recoge el principio de indisolubilidad entre la actividad de investigación y la actividad docente. Así pues, parece razonable explorar la vía de facilitar de forma más activa la integración de este profesorado en equipos de investigación relacionados con su campo de conocimiento y experiencia profesional, a modo de enriquecimiento en ambas direcciones. Algún estudio recoge el interés del profesorado a tiempo parcial en la investigación (López Damian et al., 2016), con el ánimo de obtener una creciente integración en el mundo académico. El personal asociado normalmente tiene un menor contacto con el mundo universitario y tiene limitaciones y barreras a la hora de acceder a grupos o proyectos de investigación. En la misma línea, se propone incentivar su asistencia y participación en congresos o seminarios.

\subsection{Promover estancias en otras Universidades}

El profesor asociado, por su propia idiosincrasia, suele tener menor disponibilidad para la realización de estancias en otras Universidades. Sin embargo, existen posibilidades como las excedencias, transiciones entre puestos de trabajo, vacaciones o estancias virtuales. La Universidad puede promover programas de intercambio adaptados a las circunstancias particulares de este colectivo.

\subsection{Acreditación}

Una acreditación para profesorado asociado puede servir a varios efectos. Por un lado, permitiría al profesor tener una guía sobre los aspectos a reforzar y le serviría de estímulo en su consecución y a modo de reconocimiento. Por otro lado, serviría para establecer un perfil competencial objetivo y dar forma a parte de las estrategias que estamos exponiendo. Facilitaría además el intercambio de profesionales entre universidades. Dicha acreditación 
podría ser el paso previo a un cambio de modalidad de contrato sin perder la esencia de profesor asociado colaborador a tiempo parcial pero con un menor carácter temporal.

\subsection{Favorecer la continuidad sin renunciar a la flexibilidad}

Muchos de los puntos enumerados anteriormente están orientados a una integración en el ámbito universitario. Se propone valorar la implementación de mecanismos que favorezcan la continuidad de los profesores asociados, con un doble objetivo. Por un lado, el de asentar los logros y hacerlos perdurar en el tiempo, y por otro lado rentabilizar los esfuerzos invertidos en los propios profesionales. Aquí entrarían de nuevo en juego aspectos como el criterio de asignación de asignaturas, y otros como el de consolidar una remuneración justa que incentive la permanencia ligada al desempeño y un marco jurídico que proporcione mayor estabilidad y garantías, siempre sin perder de vista el carácter de colaboración a tiempo parcial y la flexibilidad que se requiere y que caracteriza a esta figura.

\subsection{Descarga de tareas administrativas}

Los profesores asociados son a veces sometidos a gestiones puramente administrativas que no le son familiares, completamente ajenas a la docencia y a su campo de especialización. Estas tareas les resta tiempo y desvían su atención, por lo que debería considerarse el minimizar sus funciones como gestor, y en su caso proporcionarle la formación necesaria para su realización efectiva.

\section{Consideraciones finales}

En este artículo hemos apuntado ideas y propuestas de estrategias, así como reflexiones al respecto, con el propósito de mejorar el desempeño de los Profesores Asociados, principalmente a través de la mejora de sus distintas competencias y de su óptimo aprovechamiento. Muchas de estas propuestas estarán seguramente en el imaginario de la comunidad universitaria, pero conviene ponerlas de relevancia para impulsar el debate y promover las oportunas estrategias de mejora.

En definitiva, la figura de Profesor Asociado se ajustará más a los objetivos de su concepción en tanto en cuanto se aleje de ser un comodín o un recurso para paliar carencias o rellenar huecos, y se acerque a una herramienta que aporte un valor añadido en el aprendizaje y proceso de integración del alumno en el ámbito laboral y social, y que a su vez se reconozca, se apoye y se enriquezca de una forma sistemática para estimular y optimizar su utilidad. 
Futuros trabajos en esta línea pueden plantear cuestionarios a los distintos actores en distintos contextos para enriquecer nuestras aportaciones.

\section{Referencias}

Baldwin, R. G., \& Chronister, J. L. (2001). Teaching without tenure: Policies and practices for a new era. Johns Hopkins University Press, 2715 North Charles Street, Baltimore, MD 21218-4363.

Becher, T. (2001). Tribus y territorios académicos: la indagación intelectual y las culturas de las disciplinas (No. Sirsi) i9788474327571).

Borrero, M. A. F., \& Losada, S. G. (2012). El perfil del buen docente universitario. Una aproximación en función del sexo del alumnado. REDU. Revista de Docencia Universitaria, 10(2), 237-249.

Carreras Barnés, J., Escofet Roig, A., Gros Salvat, B., Imbernón, F., Mateo Andrés, J., Medina, J. L. M. M., ... \& Carrasco Calvo, S. (2006). Propuestas para el cambio docente en la universidad.

Cladellas-Pros, R., Castelló-Tarrida, A., \& Parrado-Romero, E. (2018). Satisfacción, salud y estrés laboral del profesorado universitario según su situación contractual. Revista de Salud Pública, 20, 53-59.

Crosier, D., Birch, P., Davydovskaia, O., Kocanova, D., \& Parveva, T. (2017). Modernisation of Higher Education in Europe: Academic Staff--2017. Eurydice Report. Education, Audiovisual and Culture Executive Agency, European Commission.

Ehrenberg, R. G. (2012). American higher education in transition. Journal of Economic Perspectives, 26(1), 193-216.

EURYDICE (2004). La profesión docente en Europa: perfil, tendencias y problemática. Informe IV: El atractivo de la profesión docente en el siglo XXI. Madrid: CIDE-MEC.

i Gené, J. M. (2018). Los profesores asociados" permanentes" de universidad y su peculiar encaje en la normativa laboral: Sentencia del Tribunal Supremo de 15 de febrero de 2018. Temas laborales: Revista andaluza de trabajo y bienestar social, (143), 265-297.

González González, J. M., Arquero Montaño, J. L., \& Hassall, T. (2014). Consolidación de la formación por competencias en la universidad española: Estudio de un caso. Educacion XX1, 17(2), 145-168.

López Damián, A. I., García Ponce de León, O., Pérez Mora, R., Montero Hernández, V., \& Rojas Ortiz, E. L. (2016). Los Profesores de Tiempo Parcial en las universidades públicas estatales: una profesionalización inconclusa. Revista de la educación superior, 45(180), 23-39.

Más-Torelló, O. (2011). El profesor universitario. Sus competencias y formación. Revista de Currículum y Formación del Profesorado, 15(3).

Ministerio de Ciencia, Innovación y Universidades. Subdirección General de Ordenación, Seguimiento y Gestión de las Enseñanzas Universitarias de la Secretaría General de Universidades. (2019). Datos y cifras del Sistema Universitario Español. Publicación 2018-2019. (Publicación e- 
NIP - - - . ecretar a eneral écnica del inisterio de ie ncia, nnovación y Universidades. https://www.ciencia.gob.es/stfls/MICINN/Universidades/Ficheros/Estadisticas/datosy-cifras-sue-2018-19.pdf

Osuna-Millan, N., Rosales, R., Lara-Rosano, F., \& Garza, A. A. (2020). Toward a Model of Management Processes to Support or Increase the Competitiveness of a University Professor. In Agents and Multi-agent Systems: Technologies and Applications 2019 (pp. 227-233). Springer, Singapore.

Sánchez-Caballero, D. (2019) Las universidades tiran de profesores precarios: los asociados ya son más que los titulares. Eldiario.es, 09/07/2019. Disponible en: https://www.eldiario.es/sociedad/precarizacion-universidad-profesores-contratadosfuncionarios_0_918658472.html

Umbach, P. D. (2007). How effective are they? Exploring the impact of contingent faculty on undergraduate education. The Review of Higher Education, 30(2), 91-123. 